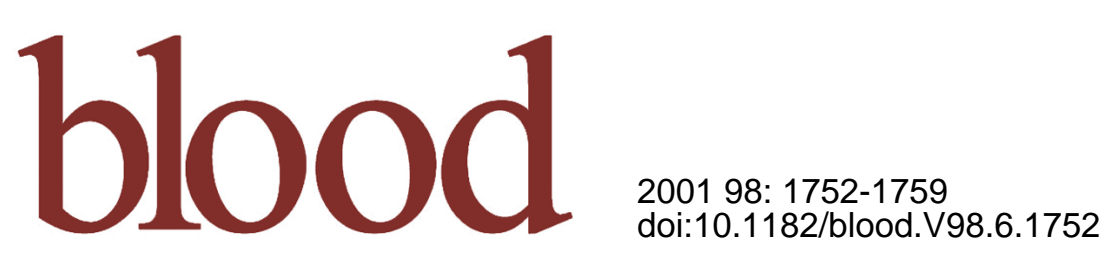

The presence of a FLT3 internal tandem duplication in patients with acute myeloid leukemia (AML) adds important prognostic information to cytogenetic risk group and response to the first cycle of chemotherapy: analysis of 854 patients from the United Kingdom Medical Research Council AML 10 and 12 trials

Panagiotis D. Kottaridis, Rosemary E. Gale, Marion E. Frew, Georgina Harrison, Stephen E. Langabeer, Andrea A. Belton, Helen Walker, Keith Wheatley, David T. Bowen, Alan K. Burnett, Anthony H. Goldstone and David C. Linch

Updated information and services can be found at: http://bloodjournal.hematologylibrary.org/cgi/content/full/98/6/1752

Articles on similar topics may be found in the following Blood collections:

Clinical Trials and Observations (2413 articles)

Neoplasia (4100 articles)

Information about reproducing this article in parts or in its entirety may be found online at: http://bloodjournal.hematologylibrary.org/misc/rights.dtl\#repub_requests

Information about ordering reprints may be found online at:

http://bloodjournal.hematologylibrary.org/misc/rights.dt|\#reprints

Information about subscriptions and ASH membership may be found online at:

http://bloodjournal.hematologylibrary.org/subscriptions/index.dtl

Blood (print ISSN 0006-4971, online ISSN 1528-0020), is published semimonthly by the American Society of Hematology, 1900 M St, NW, Suite 200, Washington DC 20036.

Copyright 2007 by The American Society of Hematology; all rights reserved.

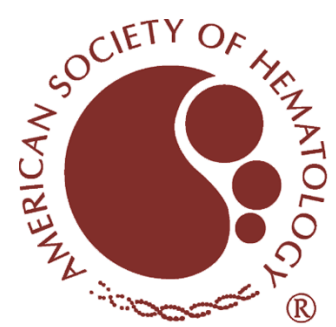




\title{
The presence of a FLT3 internal tandem duplication in patients with acute myeloid leukemia (AML) adds important prognostic information to cytogenetic risk group and response to the first cycle of chemotherapy: analysis of 854 patients from the United Kingdom Medical Research Council AML 10 and 12 trials
}

\author{
Panagiotis D. Kottaridis, Rosemary E. Gale, Marion E. Frew, Georgina Harrison, Stephen E. Langabeer, Andrea A. Belton, \\ Helen Walker, Keith Wheatley, David T. Bowen, Alan K. Burnett, Anthony H. Goldstone, and David C. Linch
}

\begin{abstract}
In acute myeloid leukemia (AML), further prognostic determinants are required in addition to cytogenetics to predict patients at increased risk of relapse. Recent studies have indicated that an internal tandem duplication (ITD) in the FLT3 gene may adversely affect clinical outcome. This study evaluated the impact of a FLT3/ITD mutation on outcome in 854 patients, mostly 60 years of age or younger, treated in the United Kingdom Medical Research Council (MRC) AML trials. An FLT3/ITD mutation was present in $27 \%$ of the patients and was associated with leukocytosis and a high percentage
\end{abstract}

of bone marrow blast cells $(P<.001$ for both). It had a borderline association with a lower complete remission rate $(P=.05)$ and a higher induction death rate $(P=.04)$, and was associated with increased relapse risk (RR), adverse disease-free survival (DFS), event-free survival (EFS), and overall survival (OS) $(P<.001$ for all). In multivariate analysis, presence of a mutation was the most significant prognostic factor predicting RR and DFS $(P<.0001)$ and was still significant for OS $(P=.009)$ and EFS $(P=.002)$. There was no evidence that the relative effect of a FLT3/ITD differed between the cytogenetic risk groups. More than one mutation was detected in $23 \%$ of $\mathrm{FLT}_{3} / \mathrm{ITD}^{+}$patients and was associated with worse OS $(P=.04)$ and EFS $(P=.07)$. Biallelic disease or partial/ complete loss of wild-type alleles was present in $10 \%$ of $\mathrm{FLT} / \mathrm{ITD}^{+}$patients. The suggestion is made that detection of a FLT3/ITD should be included as a routine test at diagnosis and evaluated for therapeutic management. (Blood. 2001;98:1752-1759)

() 2001 by The American Society of Hematology

\section{Introduction}

The past 20 to 30 years have seen significant improvements in therapy for acute myeloid leukemia (AML) such that complete remission (CR) can now be achieved in about $80 \%$ of adult patients under the age of 55 years. ${ }^{1}$ However, over half the patients achieving a $\mathrm{CR}$ will have a relapse, and the long-term survival rate is about $40 \%$. $^{1,2}$

In childhood acute lymphoblastic leukemia, risk-adapted therapy, where more intensive consolidation treatment is given to patients with poorer risk disease, has proved highly successful, ${ }^{3}$ and a similar strategy is now being used for AML. The most powerful prognostic factor in AML has been the karyotype of the leukemic cells, which predicts for both the CR rate and the relapse rate (RR) in those patients achieving a CR. ${ }^{4,5}$ Three cytogenetic risk groupsfavorable, intermediate, and adverse-are widely accepted, although there are minor differences in the precise definitions of these risk groupings among investigators. ${ }^{4,5}$ In the current United Kingdom Medical Research Council (MRC) AML12 trial, patients with favorable risk cytogenetics, which include $\mathrm{t}(15 ; 17), \mathrm{t}(8 ; 21)$ and inv(16), are not recommended for transplantation in first CR. ${ }^{6}$ The prognosis for patients with adverse cytogenetics (a complex karyotype, -5 , del $(5 q),-7$ and $3 q$ abnormalities) is so poor (5-year survival rate $<15 \%$ ) that these patients are now considered for experimental therapy.

A practical limitation of the cytogenetic risk definition is that the majority of patients have intermediate-risk disease. In an analysis of 1612 patients with cytogenetic data in the MRC AML 10 trial, 1072 (67\%) fell into this category. ${ }^{5}$ Additional prognostic factors and further prognostic stratification would be particularly useful in this group. From a practical viewpoint, and bearing in mind that most younger patients will enter CR, the most useful prognostic factors are those that predict relapse. The MRC collaborative group has devised a simple robust score based on cytogenetics and morphologic response to the first course of treatment. ${ }^{6}$ Although this can identify patients at high or low risk of relapse, it fails to discriminate the RR of standard-risk patients who comprise $51 \%$ of all patients and in whom optimum treatment remains uncertain. Therefore, additional prognostic factors that predominantly predict for relapse from CR are required.

Recent studies have shown that an internal tandem duplication (ITD) in the FLT3 gene is a frequent mutation in $\mathrm{AML}^{7}$; the alteration was
From the Departments of Haematology at University College London, London, United Kingdom; Ninewells Hospital, Dundee and University of Wales, Cardiff, United Kingdom; Clinical Trial Service Unit, University of Oxford, Oxford, United Kingdom; and Birmingham Clinical Trials Unit, University of Birmingham, Birmingham, United Kingdom.

Submitted February 8, 2001; accepted May 17, 2001.

P.D.K. and M.E.F. are supported by the Leukaemia Research Fund. We are indebted to the Kay Kendall Leukaemia Fund for supporting the MRC AML trials cytogenetics database and DNA/RNA sample bank.

Reprints: Panagiotis D. Kottaridis, Department of Haematology, University College London, 98 Chenies Mews, London WC1E 6HX, United Kingdom e-mail: p.kottaridis@ucl.ac.uk.

The publication costs of this article were defrayed in part by page charge payment. Therefore, and solely to indicate this fact, this article is hereby marked "advertisement" in accordance with 18 U.S.C. section 1734.

(c) 2001 by The American Society of Hematology 
present in 46 (23\%) of 201 patients in the largest study reported to date. ${ }^{8}$ The exact stretch of DNA duplicated varies from approximately 18 to 108 nucleotides in individual patients, but it is always in frame and occurs in the juxtamembrane region of this class III tyrosine kinase receptor. ${ }^{9}$ The receptor is expressed on early hematopoietic progenitor cells and the majority of AML blast cells,${ }^{10}$ and although the precise mechanism by which the mutated FLT3 gene contributes to the pathogenesis of leukemia is unclear, it is generally thought to involve a gain-of-function of the receptor.9,11,12

Controversy exists as to the prognostic significance of these mutations. In the Japanese study, presence of the FLT3 mutation did not appear to influence the CR rate but predicted for RR and adversely predicted for overall survival (OS). ${ }^{8}$ A subsequent relatively small Dutch study suggested that FLT3 mutations were associated with both a lower CR rate and an increased RR. ${ }^{13} \mathrm{~A}$ German study reported in abstract form has, however, shown no prognostic significance to the presence of a FLT3 mutation. ${ }^{14}$

To resolve these issues we have examined the impact of FLT3/ITD mutations on clinical outcome in a large group of well-documented patients, mostly less than 60 years of age, treated intensively in the United Kingdom MRC AML 10 and 12 trials. The median follow-up of surviving patients included in this study was
52 months, with some patients followed up for over 10 years, allowing long-term analysis of RRs and survival. In addition we have performed semiquantitative polymerase chain reaction (PCR) on FLT3/ITD ${ }^{+}$cases and have analyzed the outcome in patients with multiple mutations and with biallelic disease (or monoallelic disease with loss of wild-type [WT] alleles).

\section{Patients, materials, and methods}

\section{Patients}

DNA was available from peripheral blood (PB) or bone marrow (BM) from 854 eligible adult patients at diagnosis who had been entered into either the MRC AML $10(\mathrm{n}=406)$ or AML $12(\mathrm{n}=448)$ trials. The majority of patients $(792$ of $854,93 \%)$ had de novo AML. Median age at entry was 41 years and only 4 patients were older than 60 years of age (61-63 years). Details of the patients' age, sex, French-American-British (FAB) type, PB white blood cell (WBC) count, and percentage BM blasts are given in Table 1.

\section{Therapy}

Protocols for the AML 10 trial have been published in detail elsewhere. ${ }^{1}$ Essentially patients were randomized to receive 2 courses of induction

Table 1. Clinical and demographic characteristics of 854 AML patients

\begin{tabular}{|c|c|c|c|c|c|}
\hline & Total & $\begin{array}{c}\mathrm{FLT3} / \mathrm{ITD}^{-} \\
\text {(percent total ITD } \\
\text { ( ) }\end{array}$ & $\begin{array}{c}\text { FLT3/ITD }{ }^{+} \\
\left(\text {percent total ITD }{ }^{+} \text {) }\right.\end{array}$ & $\begin{array}{l}\text { Percent } \\
\text { FLT3/ITD }^{+}\end{array}$ & $P$ \\
\hline Total & 854 & 627 & 227 & $27 \%$ & \\
\hline AML 10 & $406(48 \%)$ & 301 (48\%) & 105 (46\%) & $26 \%$ & .7 \\
\hline AML 12 & 448 (52\%) & 326 (52\%) & 122 (54\%) & $27 \%$ & \\
\hline De novo & 792 (93\%) & $582(93 \%)$ & 210 (93\%) & $27 \%$ & .9 \\
\hline Secondary & $62(7 \%)$ & $45(7 \%)$ & $17(7 \%)$ & $27 \%$ & \\
\hline \multicolumn{6}{|l|}{ FAB type } \\
\hline Mo & $14(2 \%)$ & $14(2 \%)$ & $0(0 \%)$ & $0 \%$ & .02 \\
\hline M1 & $148(17 \%)$ & 108 (17\%) & 40 (18\%) & $27 \%$ & 1.0 \\
\hline M2 & $210(25 \%)$ & $161(26 \%)$ & 49 (22\%) & $23 \%$ & .2 \\
\hline M3 & 159 (19\%) & $101(16 \%)$ & 58 (26\%) & $36 \%$ & .004 \\
\hline M4 & $172(20 \%)$ & 121 (19\%) & 51 (22\%) & $29 \%$ & .4 \\
\hline M5 & 81 (10\%) & $61(10 \%)$ & 20 (9\%) & $25 \%$ & .7 \\
\hline M6 & $15(2 \%)$ & $14(2 \%)$ & $1(<1 \%)$ & $7 \%$ & .08 \\
\hline M7 & $11(1 \%)$ & $11(2 \%)$ & $0(0 \%)$ & $0 \%$ & .04 \\
\hline RAEB-t & $8(1 \%)$ & $7(1 \%)$ & $1(<1 \%)$ & & .7 \\
\hline Unknown & $35(4 \%)$ & $29(5 \%)$ & $6(3 \%)$ & & \\
\hline \multicolumn{6}{|l|}{ Sex } \\
\hline Male & $432(51 \%)$ & 322 (51\%) & 110 (48\%) & $25 \%$ & .5 \\
\hline Female & 422 (49\%) & 305 (49\%) & 117 (52\%) & $28 \%$ & \\
\hline \multicolumn{6}{|l|}{ Age (y) } \\
\hline Younger than 15 & $5(1 \%)$ & $3(<1 \%)$ & $2(1 \%)$ & $40 \%$ & .3 \\
\hline $15-34$ & 290 (34\%) & $222(35 \%)$ & $68(30 \%)$ & $23 \%$ & \\
\hline $35-54$ & $477(56 \%)$ & $342(55 \%)$ & 135 (59\%) & $28 \%$ & \\
\hline 55 or older & $82(10 \%)$ & $60(10 \%)$ & $22(10 \%)$ & $27 \%$ & \\
\hline Median & 41 & 41 & 42 & & .2 \\
\hline \multicolumn{6}{|l|}{ WBC count $\left(\times 10^{9} / \mathrm{L}\right)$} \\
\hline Below 10 & $300(35 \%)$ & $251(40 \%)$ & $49(22 \%)$ & $16 \%$ & $<.001$ \\
\hline $10-19$ & $132(16 \%)$ & $98(16 \%)$ & $34(15 \%)$ & $26 \%$ & \\
\hline $20-49$ & $168(20 \%)$ & $125(20 \%)$ & $43(19 \%)$ & $26 \%$ & \\
\hline Median & 18.3 & 14.3 & 38 & & $<.001$ \\
\hline \multicolumn{6}{|l|}{ Percent BM blasts } \\
\hline Below $80 \%$ & 349 (41\%) & 285 (45\%) & $64(28 \%)$ & $18 \%$ & $<.001$ \\
\hline $80 \%$ or above & 417 (49\%) & 278 (44\%) & 139 (61\%) & $33 \%$ & \\
\hline Median & 80 & 79 & 87 & & $<.001$ \\
\hline
\end{tabular}

$P$ values are for the Mantel-Haenszel test for trend in age, WBC count, percent BM blasts, and Fisher exact test for AML 10 versus AML 12 , de novo versus secondary AML and each individual FAB type versus all other known FAB types.

RAEB-t indicates refractory anemia with excess blasts in transformation. 
From www.bloodjournal.org at UCL Library Services on August 28, 2008. For personal use only.

therapy of either DAT (daunorubicin, cytarabine, 6-thioguanine): $3+10$ for course 1 and $3+8$ for course 2, or ADE (cytarabine, daunorubicin, etoposide): $10+3+5$ for course 1 and $8+3+5$ for course 2 . Both arms then received 2 courses of consolidation therapy of MACE (amsacrine, cytarabine, etoposide) and MIDAC (mitozantrone, cytarabine). Patients achieving CR were allowed to proceed to an allogeneic transplant if a suitable HLA-matched donor was available or they were eligible to be randomized to receive either an autologous transplant or no further treatment.

Patients entered into the AML 12 trial were randomized to receive one course of induction therapy of either ADE $10+3+5$ or MAE (mitozantrone, cytarabine, etoposide: $3+10+5$ ). BM remission status was then assessed and patients were assigned to one of 3 risk groups-good, standard, or poor-based on the cytogenetics and response to the first cycle of therapy. ${ }^{6}$ Patients in the good-risk and standard-risk categories received a second course of induction chemotherapy as before, either ADE $8+3+5$ or MAE $3+8+5$, then a third course of MACE consolidation therapy. Good-risk patients were then randomized to receive either just one further course of chemotherapy (MIDAC) or a fourth course of ICE (idarubicin, cytarabine, etoposide) plus a fifth course of MIDAC. Standard-risk patients were randomized to receive either MIDAC, or ICE then MIDAC, or ICE followed by a transplant, or a transplant only, in a 4 versus 5 courses and transplant versus chemotherapy $2 \times 2$ design. Patients in the transplant groups received an allogeneic transplant if a suitable matched sibling donor was available or, if not, an autologous transplant. Patients assigned to the poor-risk group were entered into the MRC trial for refractory/relapsed AML and were randomized to receive reinduction chemotherapy with either standard $10+3+5$ then $8+3+5$ or continuous ADE, and then further randomized to receive either cyclosporin A or not. ${ }^{15}$

For those patients with a clinical diagnosis of acute promyelocytic leukemia, all-trans retinoic acid, either as a short or extended course, was given in addition to the chemotherapy described above. ${ }^{16}$

\section{End points}

Complete remission was defined as a normocellular BM containing less than $5 \%$ blasts and showing evidence of normal maturation of other BM elements. PB regeneration was not a requirement, ${ }^{17}$ but $97 \%$ of cases defined as CR achieved a neutrophil count of $1 \times 10^{9} / \mathrm{L}$ and a platelet count of $100 \times 10^{9} / \mathrm{L}$. Remission failures were classified by the clinicians as either partial remission (PR, defined as 5\%-15\% blasts or $<5 \%$ blasts but a hypocellular $\mathrm{BM})$, resistant disease $(\mathrm{RD},>15 \%$ blasts in the $\mathrm{BM})$ or induction death (ID, ie, related to treatment or hypoplasia). Where the clinician's evaluation was not available, deaths within 30 days of entry were classified as ID and deaths later than 30 days after entry as RD. OS was defined as the time from diagnosis to death, and event-free survival (EFS) as the time from diagnosis to an event (either failure to achieve remission, death in first CR, or relapse), with patients not achieving remission being counted as having an event on day 1. For patients achieving CR, disease-free survival (DFS) was the time from the date of first CR to an event (death in first CR or relapse) and RR was the cumulative probability of relapse, censoring at death in CR.

\section{PCR analysis of the FLT3/ITD mutation}

Exons 11 and 12 and the intervening intron of the FLT3 gene were amplified from DNA using previously described primers $11 \mathrm{~F}$ (5'-GCAATTTAGGTATGAAAGCCAGC- $\left.3^{\prime}\right)$ and 12R (5'-CTTTCAGCATTTTGACGGCAACC$\left.3^{\prime}\right) .{ }^{8}$ Approximately $100 \mathrm{ng}$ DNA was added to a reaction mix containing $1 \times$ buffer $\left(16 \mathrm{mM}\left(\mathrm{NH}_{4}\right)_{2} \mathrm{SO}_{4}, 67 \mathrm{mM}\right.$ Tris $\left.\mathrm{HCl} \mathrm{pH} \mathrm{8.8,0.01 \%} \mathrm{Tween} 20\right)$, $1.0 \mathrm{mM} \mathrm{MgCl} 2,200 \mu \mathrm{M}$ deoxynucleoside triphosphate (dNTPs) and 10 pmol each primer in a total volume of $19 \mu \mathrm{L}$. The mixture was heated to $95^{\circ} \mathrm{C}$ for 5 minutes and held at $85^{\circ} \mathrm{C}$ while $1 \mu \mathrm{L}$ containing $0.5 \mathrm{U}$ BIOTAQ DNA polymerase (Bioline, London, United Kingdom) was added; then 30 cycles each of $95^{\circ} \mathrm{C}$ for 30 seconds, $62^{\circ} \mathrm{C}$ for 30 seconds, and $72^{\circ} \mathrm{C}$ for 30 seconds were performed, followed by 5 minutes at $72^{\circ} \mathrm{C}$. Amplified products were electrophoresed through $2 \%$ agarose gels and visualized under UV light with ethidium bromide staining. A fragment of 328 base pair (bp) was produced from WT alleles. A repeat analysis was carried out on samples with an additional band (FLT3/ITD $\left.{ }^{+}\right)$.
Semiquantitative PCR was also performed on all FLT3/ITD ${ }^{+}$samples. PCR was carried out as described above with the addition of 1 pmol primer $11 \mathrm{~F}$ end-labeled with $\gamma$-[ $\left[{ }^{32} \mathrm{P}\right]-\mathrm{ATP}(3000 \mathrm{Ci} / \mathrm{mmol}$, Amersham Pharmacia Biotech, Little Chalfont, United Kingdom) and the number of amplification cycles was reduced to 25 . Products were electrophoresed through denaturing polyacrylamide gels ( $7 \mathrm{M}$ urea, $6 \%$ polyacrylamide cross-linker ratio 37.5:1, $1 \times$ Tris-borate-EDTA), the gels were dried and exposed to Hyperfilm-MP (Amersham). Autoradiographic signals were quantified using densitometry (Hoefer Scientific Instruments, San Francisco, CA) and the level of mutant FLT3 expressed as a percentage of the total signal.

\section{Statistical methods}

The Wilcoxon 2 sample test (for continuous data), Mantel-Haenszel test for trend (for grouped continuous data), and Fisher exact test (in $2 \times 2$ tables) were used to test for differences in clinical and demographic data by FLT3/ITD positivity, number of mutations (one versus 2 or more), and percentage mutation $(<60 \%$ versus $\geq 60 \%$ ). Kaplan-Meier life tables were constructed for survival data and were compared by means of the log-rank test, ${ }^{18}$ with surviving patients being censored at May 1, 1999 (AML 10 patients) or June 1, 2000 (AML 12 patients). A complete follow-up of all surviving patients to elucidate their current status was undertaken in May 1999 (AML 10) and June 2000 (AML 12) so information on relevant events is available to the above censoring dates for the vast majority of patients, with the small number $(\mathrm{n}=11)$ of patients lost to follow-up being censored at the date they were last known to be alive. Median follow-up was 52 months (range, 11-127 months). Odds ratios were calculated from the observed minus expected number of events (O-E) and its variance, $\operatorname{var}(\mathrm{O}-\mathrm{E})$, and odds ratio plots ${ }^{19}$ were used to illustrate differences in the relative effect of FLT3/ITD between prognostic factor subgroups. Multivariate logistic regression analysis with a logit link function was used to find the factors most closely associated with $\mathrm{CR}$ rate and multivariate Cox models were used to analyze OS, EFS, DFS, and RR. Models were fitted using stepwise selection, with variables added to the model if they had a $P$ less than .01 but removed if they had a $P$ more than .05 . Because of multiple testing, the level of significance was set at $P=.01$. All $P$ values are 2-tailed.

\section{Results}

\section{Incidence of FLT3/ITD and reproducibility of PCR detection}

In the total group of 854 patients, 227 (27\%) had a FLT3/ITD mutation. There was a slight difference in the incidence observed in DNA from BM (104 of 330, 32\% FLT3/ITD ${ }^{+}$) and PB (103 of 391, $26 \%$ FLT3/ITD $\left.^{+}\right)$, but this was not significant $(P=.1)$. Of 175 patients screened independently in 2 laboratories, only 5 discrepancies occurred (3\%). Four patients were found to be FLT3/ITD ${ }^{+}$in laboratory A and negative in laboratory B; 3 of these patients had very low levels of mutant $(<2 \%$, see below) with only small duplications; the other patient was negative in laboratory A on the repeat "cold" and radioactive analyses. The fourth patient was negative in laboratory A, positive in laboratory $\mathrm{B}$; re-examination of the gels and repeat analysis of the PCR showed that this sample was positive ( $9 \%$ mutant) and had been missed in the first screen in one laboratory. In addition, DNA from PB of 100 hematologically normal individuals was studied and all were found to have only WT alleles $\left(\right.$ FLT3/ITD $\left.^{-}\right)$.

\section{Characteristics of the FLT3/ITD ${ }^{+}$patients}

Details of the presenting features of the FLT3/ITD ${ }^{+}$and FLT3/ ITD $^{-}$groups are given in Table 1. Presence of the mutation was not related to gender or age. It was found in all FAB subgroups except M0 and M7 and was found in only one patient with M6, although the numbers of patients in these groups were small. It was significantly more common in M3 (58 of $159,36 \%, P=.004$ ). FLT3/ITD $^{+}$patients altogether had significantly higher PB WBC counts at diagnosis (median count for FLT3/ITD $^{-}$patients was 
From www.bloodjournal.org at UCL Library Services on August 28, 2008. For personal use only.

Table 2. Incidence of $\mathrm{FLT3}_{3} / \mathrm{ITD}^{+}$patients in different cytogenetic risk groups and subgroups

\begin{tabular}{|c|c|c|c|c|c|}
\hline Cytogenetics & Total & FLT3/ITD ${ }^{-}$ & FLT3/ITD $^{+}$ & $\begin{array}{c}\text { Percent } \\
\text { FLT3/ITD }^{+}\end{array}$ & $P$ \\
\hline Favorable risk & 242 & 184 & 58 & 24 & \\
\hline$t(15 ; 17)$ & 133 & 84 & 49 & 37 & .002 \\
\hline$t(8 ; 21)$ & 67 & 61 & 6 & 9 & .0004 \\
\hline $\operatorname{inv}(16)$ & 42 & 39 & 3 & 7 & .003 \\
\hline Intermediate risk & 434 & 302 & 132 & 30 & \\
\hline Normal & 281 & 185 & 96 & 34 & .0001 \\
\hline $\operatorname{del}(7 q)$ & 20 & 18 & 2 & 10 & .1 \\
\hline $11 q 23$ & 18 & 18 & 0 & 0 & .006 \\
\hline+8 & 74 & 53 & 21 & 28 & .7 \\
\hline+22 & 12 & 10 & 2 & 17 & .7 \\
\hline Adverse risk & 79 & 73 & 6 & 8 & \\
\hline Complex & 44 & 43 & 1 & 2 & .00003 \\
\hline $\operatorname{del}(5 q)$ & 19 & 19 & 0 & 0 & .006 \\
\hline-5 & 16 & 16 & 0 & 0 & .02 \\
\hline-7 & 30 & 28 & 2 & 7 & .01 \\
\hline$a b n(3 q)$ & 23 & 19 & 4 & 17 & .5 \\
\hline Unknown & 99 & 68 & 31 & & \\
\hline
\end{tabular}

$P$ values are from Fisher exact test for each individual cytogenetic abnormality versus all other known karyotypes.

$14.3 \times 10^{9} / \mathrm{L}$ and for FLT3/ITD ${ }^{+}$patients $\left.38.0 \times 10^{9} / \mathrm{L}, P<.001\right)$, and this was also the case for patients with FAB type M3 (median count for FLT3/ITD ${ }^{-}$patients was $3.3 \times 10^{9} / \mathrm{L}$ and for FLT3/ITD ${ }^{+}$ patients, $\left.11.4 \times 10^{9} / \mathrm{L}, P<.0001\right)$. The percentage of BM blast cells was also higher in the FLT3/ITD ${ }^{+}$patients (median for FLT3/ITD ${ }^{-}$patients $79 \%$, FLT3/ITD $\left.{ }^{+} 87 \%, P<.001\right)$.

In accord with the finding of a high frequency of FLT3/ITD mutations in FAB type M3, the incidence of FLT3/ITD in $\mathrm{t}(15 ; 17)$ was $37 \%$ (Table 2). Lower frequencies were found in $\mathrm{t}(8 ; 21)$ and $\operatorname{inv}(16)$ (9\% and $7 \%$, respectively). It is noteworthy that a high frequency of mutations was also observed in patients with normal cytogenetics $(34 \%)$. Particularly low levels of mutations were found in 11q23 (0\%), del(5q) (0\%), and complex karyotypes (2\%).

\section{Relationship between the presence of a FLT3/ITD mutation and clinical outcome}

Response rate. The $\mathrm{CR}$ rate for the 854 patients in the present study was $82 \%$. Details of the relationship between the presence of a FLT3/ITD mutation and clinical outcome, as determined by univariate analysis, are given in Table 3. Univariate analysis showed there was borderline significance to the association between the presence of a FLT3/ITD mutation and a lower CR rate $(P=.05)$ and a higher ID rate $(P=.04)$ but no significant relationship with $\mathrm{RD}(P=.4)$. There was also no difference between those with and those without a mutation in the number of courses of therapy required to achieve CR (data not shown). On multivariate analysis, the presence of a FLT3/ITD mutation did not

Table 3. Clinical outcome in FLT3/ITD- and FLT3/ITD ${ }^{+}$patients

\begin{tabular}{lrccc}
\hline & Total & FLT3/ITD $^{-}$ & FLT3/ITD $^{+}$ & $P$ \\
\hline No. of patients & 854 & 627 & 227 & \\
CR & $82 \%$ & $84 \%$ & $78 \%$ & .05 \\
ID & $8 \%$ & $7 \%$ & $11 \%$ & .04 \\
RD & $10 \%$ & $9 \%$ & $11 \%$ & .4 \\
Outcome at 5y & & & & \\
RR & $49 \%(2.1)$ & $44 \%(2.4)$ & $64 \%(4.1)$ & $<.001$ \\
DFS & $42 \%(2.0)$ & $46 \%(2.3)$ & $30 \%(3.6)$ & $<.001$ \\
EFS & $35 \%(1.7)$ & $39 \%(2.0)$ & $23 \%(2.9)$ & $<.001$ \\
OS & $41 \%(1.8)$ & $44 \%(2.1)$ & $32 \%(3.2)$ & $<.001$ \\
\hline
\end{tabular}

$P$ values are for Mantel-Haenszel test for trend for $\mathrm{CR}$ and reasons for failure, and log-rank test for outcome at 5 years. Figures in parentheses are SEs. predict for the attainment of remission. The significant factors for increasing $\mathrm{CR}$ rates were better cytogenetic risk group $(P<.0001)$, FAB type M3 $(P<.0001)$, lower initial WBC count $(P=.0002)$, female gender $(P=.004)$, and lower age $(P=.01)$.

Survival. The overall actuarial survival at 5 years from diagnosis was $41 \%$ (SE 1.8) for all patients with FLT3/ITD data. In univariate analysis the presence of a FLT3/ITD mutation strongly predicted for RR, DFS, EFS, and OS (all $P<.001$; Figure 1). A Cox multivariate analysis was performed for RR, DFS, EFS, and OS, the variables considered being the presence of a FLT3/ITD mutation, the cytogenetic risk group, presentation WBC count, percentage BM blasts at diagnosis, age, gender, and FAB type. In addition, for analyses of outcome after CR, the response status after course 1 of therapy was included because this parameter would always be used clinically in decisions about treatment intensification. Presence of a FLT3/ITD mutation was the most significant factor adversely affecting RR $(P<.0001)$ and DFS $(P<.0001)$. Cytogenetic risk group was more significant than presence of FLT3/ITD for EFS and OS $(P<.0001)$, reflecting the additional influence of cytogenetics on the CR rate, but FLT3/ITD still added prognostic information to the other prognostic factors $(P=.009$ for OS and $P=.002$ for EFS). Figure 2 shows the risk of relapse by FLT3/ITD within risk group, cytogenetic risk, and status after course 1 subgroups. Tests for trend did not identify any subgroup of patients where the relative effect of FLT3/ITD was significantly different from the overall result. Therefore, the best estimate of the relative effect of FLT3/ITD in any of the subgroups presented is the overall result. Figure 3 shows the relapse rate in patients with or without a FLT3/ITD grouped according to risk category.

\section{Quantification and number of FLT3/ITD mutations}

Semiquantitative radioactive PCR was carried out on 224 of the $227 \mathrm{FLT} / \mathrm{ITD}^{+}$samples. This was more sensitive than agarose gel electrophoresis at separating bands with small size differences and showed that a significant proportion of patients $(51 ; 23 \%)$ had more than one FLT3/ITD mutation present (Figure 4A). The majority of these patients $(45 ; 88 \%)$ had 2 mutations but up to 4 were observed. In most patients one mutation was predominant and the other(s) were minor components, but in 15 patients the mutations were present in approximately equal proportions. There was a suggestion that patients with 2 or more mutations presented with a higher 

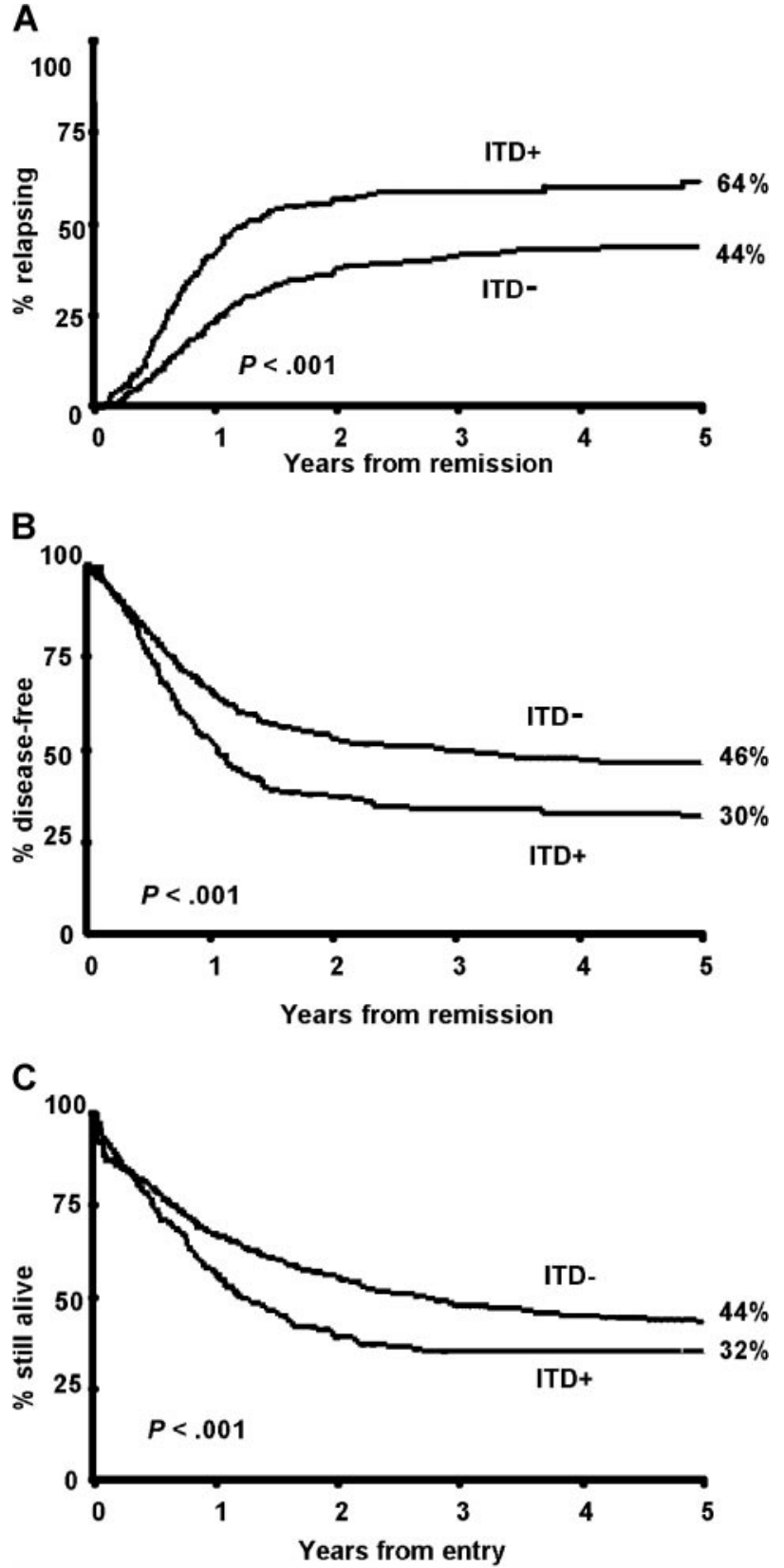

Figure 1. Kaplan-Meier curves for $\mathrm{AML}$ patients with (ITD ${ }^{+}$) or without (ITD-) a FLT3/ITD. (A) RR, (B) DFS, (C) OS.

WBC count (median WBC count for patients with one FLT3/ITD $34.4 \times 10^{9} / \mathrm{L}, 2$ or more $\left.51.5 \times 10^{9} / \mathrm{L}, P=.07\right)$ and a higher percentage of blasts in the $\mathrm{BM}$ at diagnosis (median $86 \%$ for patients with one FLT3/ITD, $90 \%$ for 2 or more, $P=.07$; Table 4$)$. Patients with 2 or more mutations had a similar RR (64\% at 5 years, SE 8.7$)$ to those with a single mutation $(64 \%, \operatorname{SE} 4.6, P=0.6)$. There was, however, a suggestion that patients with 2 or more mutations had a worse OS (36\% [SE 3.7] at 5 years for one mutation, $21 \%$ [SE 5.8] for 2 or more, $P=.04$; Figure 5A). This was due to a significantly higher number of deaths in remission in those patients with 2 or more mutations ( $34 \%$ versus $13 \%$, $P=.04)$, which may have occurred by chance. When the number of mutations ( 0 versus 1 versus 2 or more) was added into the multivariate analysis as well as the presence or absence of the mutation, then the number of mutations replaced the latter as the most important independent variable predicting for $\mathrm{RR}(P<.0001)$ and DFS $(P<.0001)$. In addition, the number of mutations became the second most important factor predicting for EFS $(P=.0007)$ and OS $(P=.0007)$, with cytogenetic risk being the most important factor $(P<.0001$ in both cases $)$.

The level of mutant detected varied between $0.5 \%$ and $97 \%$ of total FLT3 signal (Figure 4B). From previous evaluation of the relative intensity of $\mathrm{X}$-chromosome alleles using a similar technique, we have found that the proportion of signal from one allele where there are 2 equally represented alleles is $54 \% \pm 4 \%$ (Gale et al, unpublished observations). We therefore used a technical cutoff of $60 \%(50 \% \pm 2 \mathrm{SDs})$ to represent the presence of 2 abnormal alleles in a cell or the presence of a single mutant allele plus deletion of the other allele in at least some cells. Most patients (201 of $224,90 \%$ ) had less than $60 \%$ mutant. In a significant proportion of patients $(59 ; 26 \%)$ the level was consistent with a heterozygous mutation in all or the majority of cells, that is, $40 \%$ to $60 \%$ mutant. However, in 23 patients $(10 \%)$ there was $60 \%$ or more mutant, suggesting biallelic mutation or loss of WT alleles. This was associated with an older age at diagnosis; the median age at presentation in the group with less than $60 \%$ mutant was 42 years and with $60 \%$ or more mutant, 49 years; $P=.04$ (Table 4 ). It was also associated with leukocytosis; the median WBC count at diagnosis was $35.2 \times 10^{9} / \mathrm{L}$ with less than $60 \%$ mutant, $63 \times 10^{9} / \mathrm{L}$ with $60 \%$ or more mutant $(P=.03)$, poorer cytogenetic risk group $(P=.03)$, and FAB type M4 $(P=.02)$. No patients with $60 \%$ or more mutant had FAB type M3 $(P<.001)$. There was no difference in achievement of CR in the 2 groups, but there was a suggestion of worse outcome in patients with $60 \%$ or more mutant, with a marginally increased RR in this group (85\% [SE 11.9] versus $62 \%$ [SE 4.3] at 5 years, $P=.06$; Figure 5B). When the percentage mutation $(0 \%$ versus $<60 \%$ versus $\geq 60 \%)$ was added as a parameter to the multivariate analysis that already included the number of mutations, then no further prognostic information was obtained.

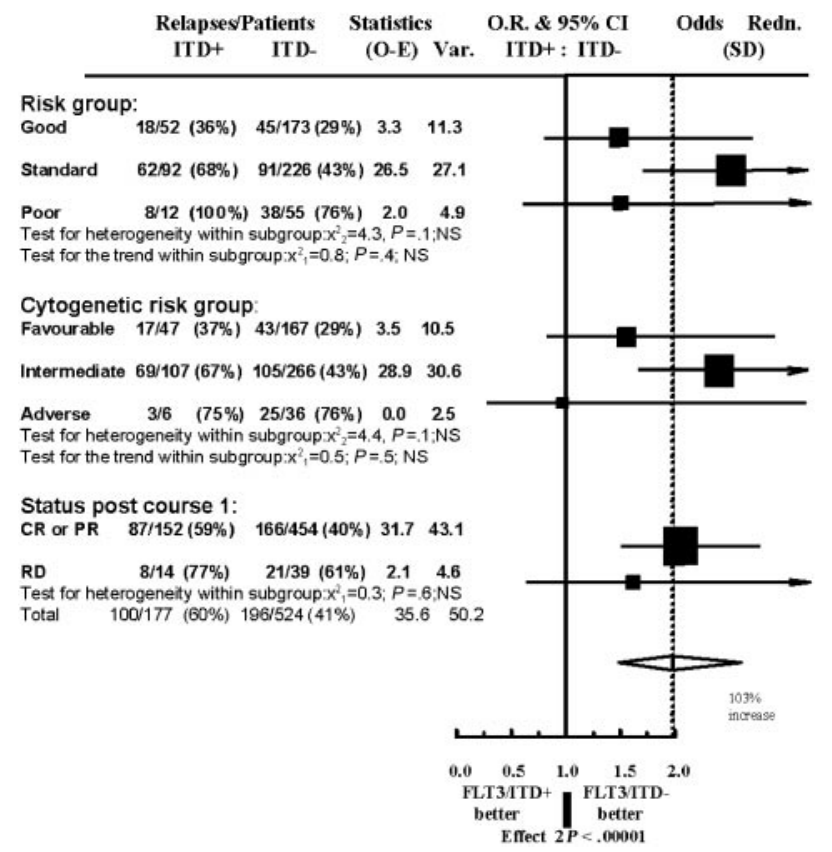

Figure 2. Relapse risk in patients with or without a FLT3/ITD. Patients are grouped according to risk category, cytogenetic risk category, or BM status after course 1 of chemotherapy. 
Figure 3. Kaplan-Meier curves showing relapse rate in patients with or without a FLT3/ITD grouped according to risk category.



\section{Discussion}

In this large study of leukemic blast cells from patients with AML we have found the incidence of FLT3/ITD mutations to be $27 \%$, which was marginally higher than that found in the Japanese (23\%), Dutch (22\%), and German (23\%) studies. ${ }^{8,13,14}$ It is possible that all studies based on DNA banks slightly overestimate the true incidence of FLT3/ITD mutations because there may be bias toward availability of DNA from patients with higher peripheral
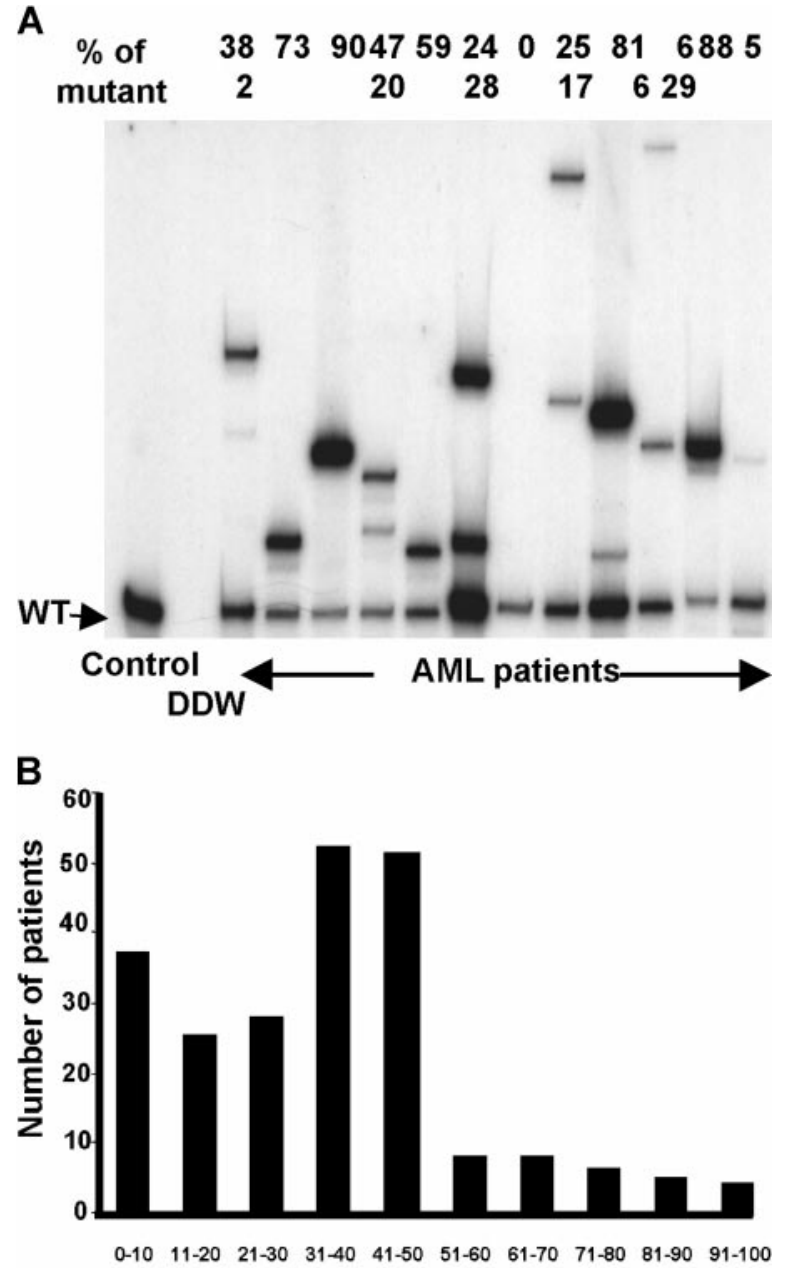

Level of mutant FLT3/ITD (\% of total FLT3)

Figure 4. Quantification and number of FLT3/ITD mutations. (A) Semiquantitative radioactive $\mathrm{PCR}$ of 12 patients with $\mathrm{AML}$. The relative percentage of the mutant(s) is shown above the gel. (B) Total level of mutant(s) detected in $224 \mathrm{FLT3} / \mathrm{ITD}^{+}$patients.
WBC counts, which we and others have shown is associated with such mutations. ${ }^{8}$ In addition, $19 \%$ of our patients had FAB type M3, which had the highest incidence of FLT3/ITD mutations (36\%) of any FAB type. The proportion of M3 cases in our study slightly exceeds the expected incidence of about $15 \%$ in this age group, ${ }^{1}$ reflecting the thoroughness of DNA collection in this disease subtype. Despite these minor reservations, it is clear that FLT3/ITD mutations are the most common single mutation described in AML.

On multivariate analysis, presence of a FLT3/ITD mutation was not found to predict for the attainment of CR, which is in accord with the Japanese and German data, although not the Dutch study. ${ }^{8,13,14}$ The lack of effect on the CR rate may be because the FLT3/ITD mutation does not significantly affect chemosensitivity of the majority of blast cells present at diagnosis, although it must be noted that as most patients achieve CR any subtle effects on chemosensitivity might not be clinically apparent as determined by


Figure 5. Outcome in FLT3/ITD ${ }^{+}$patients according to number and level of mutant(s). (A) OS in patients grouped according to the number of mutations detected. (B) RR in patients grouped according to the relative level of mutant(s). 
From www.bloodjournal.org at UCL Library Services on August 28, 2008. For personal use only.

Table 4. Clinical and demographic characteristics of patients grouped according to the number of FLT3/ITDs and the relative level of mutation

\begin{tabular}{|c|c|c|c|c|c|c|}
\hline & $\begin{array}{c}\text { One FLT3/ITD } \\
\text { (percent of total } \\
\text { with one FLT3/ITD) }\end{array}$ & $\begin{array}{c}\text { More than one FLT3/ITD } \\
\text { (percent of total with more } \\
\text { than one) }\end{array}$ & $P$ & $\begin{array}{l}\text { Less than } 60 \% \text { FLT3/ITD } \\
\text { (percent of total with } \\
<60 \% \text { ) }\end{array}$ & $\begin{array}{c}60 \% \text { or more FLT3/ITD } \\
\text { (percent of total with } \\
\geq 60 \% \text { ) }\end{array}$ & $P$ \\
\hline Total & 173 & 51 & & 201 & 23 & \\
\hline \multicolumn{7}{|l|}{ FAB type } \\
\hline M1 & $31(18)$ & $8(16)$ & .8 & $33(16)$ & $6(26)$ & .3 \\
\hline M2 & $35(20)$ & $14(27)$ & .2 & $44(22)$ & $5(22)$ & 1.0 \\
\hline M3 & $45(26)$ & $12(24)$ & .9 & $57(28)$ & $0(0)$ & .0008 \\
\hline M4 & $39(23)$ & $11(22)$ & 1.0 & $40(20)$ & $10(43)$ & .02 \\
\hline M5 & $17(10)$ & $3(6)$ & .6 & $18(9)$ & $2(9)$ & 1.0 \\
\hline M6 & $1(1)$ & $0(0)$ & 1.0 & $1(1)$ & $0(0)$ & 1.0 \\
\hline \multicolumn{7}{|c|}{ Cytogenetic risk group } \\
\hline Favorable & $44(25)$ & $12(24)$ & .3 & $56(28)$ & $0(0)$ & .03 \\
\hline Intermediate & $105(61)$ & $27(53)$ & & $116(58)$ & $16(70)$ & \\
\hline Adverse & $2(1)$ & $4(8)$ & & $6(3)$ & $0(0)$ & \\
\hline Unknown & $22(13)$ & $8(16)$ & & $23(11)$ & $7(30)$ & \\
\hline \multicolumn{7}{|l|}{ Age at entry } \\
\hline Younger than 15 & $1(1)$ & $1(2)$ & .8 & $2(1)$ & $0(0)$ & .5 \\
\hline $15-34$ & $50(29)$ & $15(29)$ & & $61(30)$ & $4(17)$ & \\
\hline $35-54$ & $105(61)$ & $30(59)$ & & $117(58)$ & $18(78)$ & \\
\hline 55 or older & $17(10)$ & $5(10)$ & & $21(10)$ & $1(4)$ & \\
\hline Median & 43 & 41 & .3 & 42 & 49 & .04 \\
\hline \multicolumn{7}{|l|}{ WBC count $\left(\times 10^{9} / L\right)$} \\
\hline Below 10 & $40(23)$ & $7(14)$ & .1 & $46(23)$ & $1(4)$ & .3 \\
\hline $10-19$ & $29(17)$ & $5(10)$ & & $31(15)$ & $3(13)$ & \\
\hline $20-49$ & $32(19)$ & $10(20)$ & & $38(19)$ & $4(17)$ & \\
\hline $50-99$ & $32(19)$ & $12(24)$ & & $34(17)$ & $10(43)$ & \\
\hline 100 or above & $39(23)$ & $14(27)$ & & $48(24)$ & $5(22)$ & \\
\hline Median & 34.4 & 51.5 & .07 & 35.2 & 63.0 & .03 \\
\hline \multicolumn{7}{|l|}{ Percent BM blasts } \\
\hline Below $80 \%$ & $54(31)$ & $10(20)$ & .2 & $56(28)$ & $8(35)$ & .6 \\
\hline $80 \%$ or above & $104(60)$ & $33(65)$ & & $123(61)$ & $14(61)$ & \\
\hline Median & 86 & 90 & .07 & 86 & 87.5 & .5 \\
\hline \multicolumn{7}{|l|}{ Sex } \\
\hline Male & $80(46)$ & $28(55)$ & .3 & $102(51)$ & $6(26)$ & .3 \\
\hline Female & $93(54)$ & $23(45)$ & & $99(49)$ & $17(74)$ & \\
\hline
\end{tabular}

$P$ values are Mantel-Haenszel test for the trend in cytogenetic risk, age, WBC count, percent BM blasts, and Fisher exact test for each individual FAB type versus all othe known FAB types.

the CR rate. The presence of a FLT3/ITD mutation did, however, have a major impact on long-term outcome in our cohort of patients. In particular, it was the most important factor predicting for relapse from CR $(P<.0001)$, and in the presence of a FLT3 mutation the actuarial RR at 5 years was $64 \%$ (SE 4.1) compared to $44 \%$ (SE 2.4) in patients without a mutation. The relative effect of FLT3/ITD on RR was not found to differ significantly between risk groups. The effect was most reliably demonstrable in the intermediate-risk group, whether defined by cytogenetics alone or by the combination of cytogenetics and response to the first cycle of induction chemotherapy, which is the very group in whom improved prognostic stratification is most required (Figures 2 and 3).

In accord with being the major factor predicting for relapse, the presence of a FLT3/ITD was also the major factor predicting for DFS $(P<.0001)$. Furthermore, it had an adverse effect on EFS $(P<.001)$ and OS $(P<.001)$ (Figure 1$)$, although in multivariate analysis it was considerably less significant than cytogenetic risk group for these 2 parameters because cytogenetics influences the attainment of remission as well as the subsequent RR.

It is noteworthy that within the good-risk cytogenetic group there are distinct biologic entities and they showed marked variation in the incidence of FLT3 mutations. The frequency was high in $\mathrm{t}(15 ; 17)$ at $36 \%$, and low in $\mathrm{t}(8 ; 21)$ and inv(16) at $9 \%$ and $7 \%$, respectively. It is possible that the clinical impact of a FLT3/ITD also varies between these different cytogenetic entities. However, although this is the largest study of FLT3 mutations reported to date, there are still too few patients with $\mathrm{t}(8 ; 21)$ or inv(16) and a FLT3/ITD to define their prognosis with any certainty.

The higher RR in the presence of a FLT3/ITD mutation, but the lack of an obvious effect on the $\mathrm{CR}$ rate, raises the possibility that this mutation has its greatest functional effect on the small population of clonogenic cells presumed to be responsible for leukemic relapse..$^{20}$ The precise consequence of a mutant FLT3 receptor is not known. Constitutive signaling in the absence of ligand may result in reduced apoptosis of the leukemic cells or may stimulate or allow increased repair capacity following cell damage; either of these mechanisms could be considered as inducing chemoresistance. Alternatively, it is possible that there is no direct causal link between the FLT3/ITD and relapse, and that both factors are due to a third factor such as an underlying form of genetic instability that might predispose to this type of mutation and also to relapse. ITDs of other genes have been reported in AML, for example, in the AML1 gene, although not at the same frequency as in the FLT3 gene, ${ }^{21,22}$ and it is possible that ITDs in as yet unknown genes may have a major effect on the pathogenesis of leukemia.

The observation that $23 \%$ of FLT3/ITD ${ }^{+}$patients had more than one FLT3/ITD mutant of varying levels (Figure 4A), with 3 or 4 mutations being detected in 6 patients, suggests that, at least in some patients, the mutations were present in different clones. In a separate study of 8 patients with more than one mutation, we have 
From www.bloodjournal.org at UCL Library Services on August 28, 2008. For personal use only.

sequenced each mutant band ( 2 or 3 ) within the same patient and found that all were in frame in exon 11 and were unrelated, indicating that multiple bands were unlikely to be due to "clonal evolution" (data not shown). Patients with 2 or more mutants did not have a significantly higher RR than those patients with just one mutation $(P=.6)$, although on multivariate analysis the number of mutations (none, one, or more than one) replaced the presence or absence of a FLT3/ITD as the most powerful predictor of RR and DFS. Patients with more than one mutation had a significantly higher mortality in remission $(P=.04)$ and this translated into a marginally reduced EFS $(P=.07)$ and OS $(P=.04)$ on univariate analysis (Figure 5A), with the number of mutations becoming the second most important factor predicting for EFS and OS in the multivariate analysis. The reason patients with more than one FLT3/ITD should have had a higher treatment-related mortality is not known and it is possible that this is a spurious association due to the play of chance. It would certainly be important to corroborate this unexpected finding in other series.

In addition to the frequent detection of more than one FLT3/ITD mutation, biallelic mutations or deletion of WT alleles were present in at least the $10 \%$ of patients where the level of mutant was $60 \%$ or more of the total FLT3 signal (Figure 4B). This $10 \%$ value is undoubtedly an underestimate, because the presence of residual normal cells in some samples (especially from PB) would tend to decrease the ratio of the mutant to WT FLT3 allele. There was a suggestion of higher RR in those patients with $60 \%$ or more mutant $(P=.06$; Figure $5 \mathrm{~B})$, but on multivariate analysis that already included the number of mutations, the presence of $60 \%$ or more mutant was not an independent prognostic factor.
The discovery that FLT3/ITDs are the most common mutations in AML raises 2 other issues besides prognosis. First, the presence of a mutation that activates a tyrosine kinase receptor introduces the possibility of using specific kinase inhibitors to treat this disease, analogous to the use of STI 571 in chronic myeloid leukemia. ${ }^{23}$ Second, a FLT3/ITD mutation might serve as a suitable marker for detection of minimal residual disease, although more extensive analysis of matched presentation and relapse samples would be needed before this could be applied clinically.

In AML, relapse remains the principal cause of treatment failure for the majority of patients. Therefore the ability to identify those patients with a high risk of relapse would represent a valuable advance, which may lead to the introduction of alternative forms of therapy in this group of patients. In a large cohort of patients we have confirmed that the presence of a FLT3/ITD is the most important factor predicting for relapse and DFS and is, as a consequence, an independent risk factor for EFS and OS. The laboratory test to identify the presence or absence of a FLT3/ITD mutation is simple, quick, and reproducible. Our study suggests that even further prognostic stratification may be achieved by considering the number of mutations and the relative ratio of the mutant and WT alleles, but this requires radioactive PCR analysis. We do not, however, believe that this additional information justifies the widespread introduction of quantitative technology, and advocate that simple "cold PCR" analysis for FLT3 mutations should now be considered as part of the routine work-up of patients with AML at diagnosis, with a view to introducing this parameter into risk stratification and patient management.

\section{References}

1. Hann IM, Stevens RF, Goldstone AH, et al. Randomized comparison of DAT versus ADE as induction chemotherapy in children and younger adults with acute myeloid leukemia. Results of the Medical Research Council's 10th AML trial (MRC AML10). Adult and Childhood Leukaemia Working Parties of the Medical Research Council. Blood. 1997;89:2311-2318.

2. Lowenberg B, Downing JR, Burnett A. Acute myeloid leukemia. N Engl J Med. 1999;341:10511062.

3. Reiter A, Schrappe M, Ludwig WD, et al. Chemotherapy in 998 unselected childhood acute lymphoblastic leukemia patients. Results and conclusions of the multicenter trial ALL-BFM 86. Blood. 1994;84:3122-3133.

4. Bloomfield CD, Shuma C, Regal L, et al. Longterm survival of patients with acute myeloid leukemia: a third follow-up of the Fourth International Workshop on Chromosomes in Leukemia. Cancer. 1997;80:2191-198.

5. Grimwade D, Walker $\mathrm{H}$, Oliver $\mathrm{F}$, et al. The importance of diagnostic cytogenetics on outcome in AML: analysis of 1,612 patients entered into the MRC AML 10 trial. The Medical Research Council Adult and Children's Leukaemia Working Parties. Blood. 1998;92:2322-2333.

6. Wheatley K, Burnett AK, Goldstone AH, et al. A simple, robust, validated and highly predictive index for the determination of risk-directed therapy in acute myeloid leukaemia derived from the MRC AML 10 trial. United Kingdom Medical Research Council's Adult and Childhood Leukaemia Working Parties. Br J Haematol. 1999;107: 69-79.

7. Nakao M, Yokota S, Iwai T, et al. Internal tandem duplication of the flt3 gene found in acute myeloid leukemia. Leukemia. 1996;10:1911-1918.
8. Kiyoi H, Naoe T, Nakano Y, et al. Prognostic implication of FLT3 and N-RAS gene mutations in acute myeloid leukemia. Blood. 1999;93:30743080.

9. Kiyoi H, Towatari M, Yokota S, et al. Internal tandem duplication of the FLT3 gene is a novel modality of elongation mutation which causes constitutive activation of the product. Leukemia. 1998; 12:1333-1337.

10. Drexler HG. Expression of FLT3 receptor and response to FLT3 ligand by leukemic cells. Leukemia. 1996;10:588-599.

11. Fenski R, Flesch K, Serve S, et al. Constitutive activation of FLT3 in acute myeloid leukaemia and its consequences for growth of 32D cells. Br J Haematol. 2000;108:322-330.

12. Hayakawa F, Towatari M, Kiyoi H, et al. Tandem duplicated Flt3 constitutively activates STAT5 and MAP kinase and introduces autonomous cell growth in IL-3-dependent cell lines. Oncogene. 2000;19:624-631.

13. Rombouts WJ, Blokland I, Lowenberg B, Ploemacher RE. Biological characteristics and prognosis of adult acute myeloid leukemia with internal tandem duplications in the Flt3 gene. Leukemia. 2000;14:675-683.

14. Schnittger S, Schoch C, Kern W, et al. Flt3 length mutations in AML: correlation to cytogenetics, FAB-subtype, and prognosis in 652 patients. Blood. 2000;96:826a.

15. Yin JAL, Wheatley K, Rees J, Burnett A. Comparison of two chemotherapy regimens, with or without cyclosporin $A$, in relapsed/refractory acute myeloid leukaemia: results of the UK Medical Research Council. Blood. 1998:92:231a.

16. Burnett AK, Grimwade D, Solomon E, Wheatley $\mathrm{K}$, Goldstone AH. Presenting white blood cell count and kinetics of molecular remission predict prognosis in acute promyelocytic leukemia treated with all-trans retinoic acid: result of the randomized MRC trial. Blood. 1999:93:4131. 4143.

17. Cheson BD, Cassileth PA, Head DR, et al. Report of the National Cancer Institute-sponsored workshop on definitions of diagnosis and response in acute myeloid leukemia. J Clin Oncol. 1990;8: 813-819.

18. Peto R, Pike MC, Armitage $P$, et al. Design and analysis of randomized clinical trials requiring prolonged observation of each patient. II. analysis and examples. Br J Cancer. 1977;35:1-39.

19. Antiplatelet Trialists' Collaboration. Secondary prevention of vascular disease by prolonged antiplatelet treatment. Br Med J. 1988;296:320-331.

20. Bonnet D, Dick JE. Human acute myeloid leukemia is organized as a hierarchy that originates from a primitive hematopoietic cell. Nat Med. 1997;3:730-737.

21. Preudhomme C, Warot-Loze D, Roumier C, et al. High incidence of biallelic point mutations in the runt domain of the AML1/PEBP2alphaB gene in M0 acute myeloid leukemia and in myeloid malignancies with acquired trisomy 21. Blood. 2000; 96:2862-2869.

22. Langabeer SE, Gale RE, Rollison SJ, Morgan GJ, Linch DC. Mutations in the AML1 gene in acute myeloid leukaemia patients with FAB types M0 and M7. Blood. 2000;96:698a.

23. Druker BJ, Lydon NB. Lessons learned from the development of an abl tyrosine kinase inhibitor for chronic myelogenous leukemia. J Clin Invest. 2000;105:3-7. 